\title{
An Evaluation of Marginal Maximum Likelihood Estimation for the Two-Parameter Logistic Model
}

\author{
Fritz Drasgow \\ University of Illinois
}

The accuracy of marginal maximum likelihood estimates of the item parameters of the two-parameter logistic model was investigated. Estimates were obtained for four sample sizes and four test lengths; joint maximum likelihood estimates were also computed for the two longer test lengths. Each condition was replicated 10 times, which allowed evaluation of the accuracy of estimated item characteristic curves, item parameter estimates, and estimated standard errors of item parameter estimates for individual items. Items that are typical of a widely used job satisfaction scale and moderately easy tests had satisfactory marginal estimates for all sample sizes and test lengths. Larger samples were required for items with extreme difficulty or discrimination parameters. Marginal estimation was substantially better than joint maximum likelihood estimation. Index terms: Fletcher-Powell algorithm, item parameter estimation, item response theory, joint maximum likelihood estimation, marginal maximum likelihood estimation, two-parameter logistic model.

In 1968 Lord introduced a method for estimating the parameters of the three-parameter logistic model. He reported that samples of 1,000 examinees responding to 50 items were needed for adequate estimation. A great deal of work on estimation of parameters of various item response theory (IRT) models has occurred since Lord's paper. The developments in this area have been impressive; Thissen (1982), for example, stated that "the general problem of item parameter estimation in item response theory has been effectively solved" (p. 186).

Lord's (1968) approach to estimation can be called joint maximum likelihood (JML) estimation because the person parameter $(\theta)$ and the item parameters are estimated simultaneously. To write Lord's likelihood function, let $U_{i j}$ denote the dichotomous response of person $i(i=1, \ldots, N)$ to item $j(j=1, \ldots, n)$, where $U_{i j}=0$ or $U_{i j}=1$ depending on whether the response is incorrect or correct, and let $P_{j}\left(\theta_{i}\right)=$ $P\left(U_{i j}=1 \mid \theta_{i}\right)$ denote the probability of a correct response by examinee $i$ with ability $\theta_{i}$. The item parameters and the functional form of $P_{j}\left(\theta_{i}\right)$ depend on the item response model. If the local independence and examinee independence assumptions are met, the joint distribution of the $N \times n$ matrix $U$ of item responses is

$\mathbf{L}=\prod_{i=1}^{N} \prod_{j=1}^{n} P_{j}\left(\theta_{i}\right)^{u_{j}}\left[1-P_{j}\left(\theta_{i}\right)\right]^{u_{i j}}$.

Lord's likelihood function is Equation 1 viewed as a function of the item parameters and the $\boldsymbol{\theta}_{i}$.

APPLIED PSYCHOLOGICAL MEASUREMENT

Vol. 13, No. 1, March 1989, pp. 77-90

(C) Copyright 1989 Applied Psychological Measurement Inc.

0146-6216/89/010077-14\$1.95 
Suppose that a test of fixed length is administered to a large sample, such as a national administration of the Scholastic Aptitude Test verbal section. No additional item parameters are needed as the number $N$ of examinees used for item calibration is increased. Moreover, each item parameter appears in the distribution of a large number of item responses. In this situation the item parameters are said to be structural. In contrast, an additional $\theta$ is needed for each examinee added to the sample. The $\theta$ of a particular examinee appears in the distribution of only $n$ item responses and, consequently, is said to be an incidental parameter. For a test of fixed length, asymptotic properties (e.g., consistency) obviously cannot hold for maximum likelihood estimates (MLEs) of the $\theta$ s. Interestingly, Neyman and Scott (1948) showed that MLEs of structural parameters are not necessarily consistent when incidental parameters are present. Thus, MLEs of item parameters obtained from Equation 1 may not have the usual properties of MLES.

Computer simulations (e.g., Hulin, Lissak, \& Drasgow, 1982) have shown that parameter estimates obtained by maximizing Equation 1 are quite accurate under the conditions specified by Lord (1968). Many situations exist, however, in which researchers have fewer than 50 items and 1,000 examinees. For example, the Job Descriptive Index (JDI; Smith, Kendall, \& Hulin, 1969) is widely used to assess five approximately unidimensional aspects of job satisfaction. Each JDI scale consists of either 9 or 18 items and researchers commonly collect data from 200 to 400 persons.

It is important to have accurate item parameter estimates for applications of IRT to scales similar to the JDI that have limited numbers of items and are used with relatively small samples. In this paper, the use of marginal maximum likelihood (MML) estimation is examined for this problem.

Maximum likelihood estimation of structural parameters when incidental parameters are present was studied by Kiefer and Wolfowitz (1956). Rather than estimating the values of the incidental parameters (as Lord's method estimates $\theta \mathrm{s}$ ), Kiefer and Wolfowitz assumed that the incidental parameters were sampled from an unknown distribution $G$. Let $x_{i}$ denote the data for the $i$ th observation and let $\boldsymbol{\beta}$ denote the structural parameters. Kiefer and Wolfowitz's marginal density of the data given $\boldsymbol{\beta}$ and $G$ is

$f\left(x_{i} \mid \boldsymbol{\beta}, G\right)=\int f\left(x_{i} \mid \boldsymbol{\beta}, t\right) d G(t)$,

where $t$ is integrated over the range of possible values of the incidental parameter. Let $\Omega$ denote the space of values of $\boldsymbol{\beta}$, and let $\Gamma$ denote the space of possible distribution functions $G$. The product over observations of the $f\left(x_{i} \mid \boldsymbol{\beta}, G\right)$ viewed as a function of the points in $\Omega \times \Gamma$ constitutes Kiefer and Wolfowitz's likelihood function. They showed that the MLEs of the structural parameters obtained from this likelihood function were strongly consistent.

Bock and Lieberman (1970) used the idea of removing incidental parameters from the likelihood function through integrating in the context of IRT. They simplified Kiefer and Wolfowitz's approach by assuming that $\theta$ s were sampled from a normal distribution. The marginal likelihood of the $i$ th examinee's responses is then

$\mathrm{L}_{i}^{*}=\int\left\{\prod_{j=1}^{n} P_{j}(\theta)^{u_{\eta}}\left[1-P_{j}(\theta)\right]^{1-u_{\eta}}\right\} \phi(\theta) d \theta$,

where $\phi(\cdot)$ is the standard normal density, and the overall likelihood function is

$\mathrm{L}^{*}=\prod_{i=1}^{N} \mathrm{~L}_{i}^{*}$.

By taking Kiefer and Wolfowitz's $G$ to be the standard normal, Bock and Lieberman reduced the problem of maximizing a likelihood function in $\Omega \times \Gamma$ to maximizing only in $\Omega$. As an alternative to assuming a normal $\theta$ density, Bock and Aitkin (1981) characterized the $\theta$ density by a discrete distribution on a 
prespecified number of points, and then jointly estimated the $\theta$ distribution and item parameters. Mislevy (1986) also studied joint estimation of item parameters and the $\theta$ distribution, although he used Bayesian estimation rather than maximum likelihood.

Since its introduction by Bock and Lieberman (1970), MML estimation has been applied to many IRT models (e.g., Bock \& Aitkin, 1981; Mislevy \& Bock, 1985; Thissen, 1982; Thissen \& Steinberg, 1984). BILOG (Mislevy \& Bock, 1985) and MULTILOG (Thissen, 1986) are computer programs that provide MML estimates (MMLEs) for a variety of IRT models. These programs greatly reduce the computational burden for long tests through the use of the EM algorithm (Dempster, Laird, \& Rubin, 1977).

\section{Overview of the Present Research}

MML estimation for the two-parameter logistic model was studied because this model provides a reasonably good fit to JDI data (Hulin, Drasgow, \& Parsons, 1983). Tests of 5 to 25 items and samples of 200 to 1,000 simulated examinees were examined because these ranges encompass the values seen in many applied studies. Ten replications per cell were used in the simulation.

Although they have been studied far less frequently than item parameter estimates, estimates of standard errors of parameter estimates are crucial for many practical applications of IRT. For example, McLaughlin and Drasgow (1987) examined the use of Lord's (1980) Equations 12-8, 12-9, and 12-11 to estimate standard errors when item parameters and person parameters were estimated by JML estimation. They found that standard errors were consistently underestimated, which led to substantial inflation in the Type I error rate for Lord's (1980) test of item bias. Consequently, the accuracy of one approach to estimating standard errors of MMLES was evaluated in this study.

\section{Method}

\section{Program for Marginal Maximum Likelihood Estimation}

A FORTRAN program was written to estimate the $a_{j}$ (discrimination) and $b_{j}$ (difficulty) parameters of the two-parameter logistic model,

$P_{j}\left(\theta_{i}\right)=\left\{1+\exp \left[-D a_{j}\left(\theta_{i}-b_{j}\right)\right]\right\}^{-1}$,

where $D=1.702$. The program uses the dichotomously scored responses of $N$ individuals to $n$ items. Starting values for the $j$ th item were obtained by first computing the proportion $\hat{p}_{j}$ of positive responses, the biserial correlation $r_{j}$ between item $j$ and the number-correct score on the $(n-1)$-item subtest formed by excluding item $j$, and coefficient alpha for the $(n-1)$-item subtest. Then a "corrected" biserial $r_{j}^{*}$ was computed by dividing $r_{j}$ by the square root of the $(n-1)$-item subtest coefficient alpha. Restricting $r_{j}^{*}$ to the interval $[.025, .925]$ reduced problems with convergence. Starting values were obtained from

$a_{\mathrm{start}}=\frac{r_{j}^{*}}{\left[1-\left(r_{j}^{*}\right)^{2}\right]^{1 / 2}}$

and

$b_{\text {start }}=\frac{\Phi^{-1}\left(\hat{p}_{j}\right)}{r_{j}^{*}}$,

where $\Phi^{-1}(\cdot)$ is the inverse of the cumulative normal $(0,1)$ distribution function. Equations 6 and 7 are motivated by Equations 16.8.5 and 16.9.3 in Lord and Novick (1968).

The likelihood function was then maximized by adapting the function minimization routines written by Gruvaeus and Jöreskog (1970). Maximization begins with a few steepest-ascent iterations and then 
switches to Fletcher-Powell (1963) iterations. Gauss-Hermite quadrature with 20 quadrature points was used to evaluate the integrals in the expressions for the logarithm of the likelihood function and its derivatives.

The $2 n \times 2 n$ matrices of second derivatives were examined for a number of real and artificial datasets. The second derivatives were computed both at the starting values and at the maximum of the likelihood function. Second derivatives of the form $\partial^{2} \log \mathrm{L} / \partial a_{j}^{2}, \partial^{2} \log \mathrm{L} / \partial b_{j}^{2}$, and $\partial^{2} \log \mathrm{L} / \partial a_{j} \partial b_{j}$ were usually 10 to 50 times larger in absolute value than the remaining second derivatives. Consequently, the starting value of the Fletcher-Powell weight matrix was obtained by evaluating $\partial^{2} \log \mathrm{L} / \partial a_{j}^{2}, \partial^{2} \log \mathrm{L} / \partial b_{j}^{2}$, and $\partial^{2} \log \mathrm{L} / \partial a_{j} \partial b_{j}(j=1, \ldots, n)$ after the steepest-ascent iterations, setting all other second derivatives to 0 , and inverting this approximation to the second-derivative matrix.

Fletcher-Powell iterations were continued until all first derivatives were less than .0001 in absolute value. Parameter estimates usually converged (to four decimal places) several iterations before the first derivatives satisfied the convergence criterion. However, extra iterations were required for the sampling variances to stabilize. Usually 15 to 25 Fletcher-Powell iterations were required for convergence.

Occasionally a Fletcher-Powell iteration moved to a point at which parameter values updated by the Fletcher-Powell method reduced the likelihood function. At such points the Fletcher-Powell algorithm cannot continue. Consequently, several steepest-ascent iterations were performed, the Fletcher-Powell weight matrix was reinitialized to an identity matrix, and then Fletcher-Powell iterations were started again. This heuristic adaptation of Gruvaeus and Jöreskog's program worked well; execution was never prematurely terminated due to a reduced likelihood function.

\section{Item Parameters, Test Lengths, and Sample Sizes}

All item responses were generated according to the two-parameter logistic model: Item characteristic curves were two-parameter logistic ogives, the $\theta$ parameter was unidimensional, item responses were locally independent, and simulated examinees were independent. Probabilities of correct responses were computed by Equation 5, and compared to a random number sampled from a uniform [0,1] distribution; a simulated response was scored as correct if the sampled number was less than or equal to the probability of a correct response.

The item parameters used in the simulation study are listed in Table 1 . These parameter values were selected after inspecting a plot of parameter estimates obtained by Drasgow and Hulin (in press) for 486

Table 1

\begin{tabular}{crrrrr}
\multicolumn{7}{c}{ Table 1} \\
\hline \multicolumn{7}{c}{ Item } & \multicolumn{1}{c}{ Parameters Used in the Simulation Study } \\
\hline Item & \multicolumn{1}{c}{$a_{j}$} & \multicolumn{1}{c}{$b_{j}$} & Item & \multicolumn{1}{c}{$a_{j}$} & $b_{j}$ \\
\hline 1 & .80 & -1.50 & 14 & 1.40 & -1.00 \\
2 & 1.40 & -1.50 & 15 & 1.10 & .00 \\
3 & 1.10 & -1.00 & 16 & .40 & -1.00 \\
4 & .80 & .00 & 17 & 1.80 & -1.00 \\
5 & 1.40 & .00 & 18 & .40 & 1.00 \\
6 & .40 & -1.50 & 19 & 1.80 & 1.00 \\
7 & 1.80 & -1.50 & 20 & 1.10 & 1.00 \\
8 & .40 & 2.50 & 21 & .40 & -2.00 \\
9 & 1.10 & 2.50 & 22 & .80 & -2.00 \\
10 & 1.40 & 2.00 & 23 & 1.40 & -2.00 \\
11 & 1.10 & -2.00 & 24 & .40 & -.50 \\
12 & 1.10 & -1.50 & 25 & 1.10 & -.50 \\
13 & .80 & -1.10 & & & \\
\hline
\end{tabular}


persons responding to the five JDr scales. About $60 \%$ of the parameter estimates were contained in a rectangle formed by the points $(.8,-1.5),(1.4,-1.5),(.8,0.0)$, and $(1.4,0.0)$; thus, these points were used as parameter values for items $1,2,4$, and 5. The parameter values of item 3 were selected to lie in the most densely populated area of the rectangle. Parameter values of items 6 and 7 were selected to lie on the line defining the bottom of the rectangle, but to have somewhat more extreme $a_{j}$ values. Items 8,9 , and 10 had parameter values that were deliberately selected to be difficult to estimate; $b_{10}$ was originally set equal to 2.5 , but preliminary analyses encountered substantial problems in obtaining parameter estimates. Parameter values for the remaining items were selected to cover the range of JDI parameter estimates.

Table 1 clearly indicates that the item difficulties were not centered around the mean of the $\theta$ distribution. Instead, they corresponded to the item difficulties of a set of work satisfaction scales that are widely used in the United States and throughout the world (see Hulin, 1987). The item discrimination and difficulty parameters studied here also represent moderately easy tests such as the Paragraph Comprehension and Word Knowledge subtests of the Armed Services Vocational Aptitude Battery (see Mislevy \& Bock, 1984, for parameter estimates).

Although the generality of results from a simulation study is always open to question, it seems that estimation accuracy would be improved by more closely matching the distribution of difficulty parameters to the $\theta$ distribution.

Four test lengths were investigated: $n=5, n=10, n=15$, and $n=25$. Two different sets of items were used for the 5-item tests. First, items 1 through 5 simulated a 5 -item scale composed of items with typical parameter values. Then items 6 through 10 simulated a scale with difficult-to-estimate items. Items 1 through 10 were used for the 10-item test, the first 15 items were used for the 15 -item test, and all of the items were used for the 25 -item test. Ten replications of each of four sample sizes were generated. The sample sizes were $N=200, N=300, N=500$, and $N=1,000$. In all cases the response vectors were created independently.

\section{Estimation of Item Parameters}

MMLEs were computed for all datasets. A maximum of 40 Fletcher-Powell iterations was allowed. When the full 40 iterations were used, one (or more) of the item parameter estimates was very large in magnitude.

In order to provide a frame of reference for interpretation of the MMLEs, the LOGIST computer program (Wingersky, Barton, \& Lord, 1982) was also used for two-parameter logistic analyses of the 15- and 25item tests. The maximum value allowed for the $a_{j}$ parameter was set to 9.0 so that LOGIST estimates were very nearly joint maximum likelihood estimates (JMLEs). (Of course, in practical applications a user should not override LOGIST's default value for $a_{j}$ and use an absurdly large value like 9.) The maximum number of iterations allowed was $40 ; 10$ to 20 iterations were normally required. The maximum number of iterations was reached only when one or more of the $\hat{b}_{j}$ was becoming very large or when one or more of the $\hat{a}_{j}$ had reached 9 .

\section{Evaluation of Item Parameter Estimates}

Two criteria were used to evaluate item parameter estimates. Items were examined individually, across replications, for both criteria. This approach contrasts with previous studies (Hulin et al., 1982) that analyzed estimation accuracy across items for a single replication.

Let $\hat{a}_{j}^{(k)}$ and $\hat{b}_{j}^{(k)}$ denote estimates obtained on the $k$ th replication. Then the biases of the estimates of $a$, and $b$, are 
Bias of $\hat{a}_{j}=\frac{1}{10} \sum_{k=1}^{10}\left(\hat{a}_{j}^{(k)}-a_{j}\right)$

and

Bias of $\hat{b}_{j}=\frac{1}{10} \sum_{k=1}^{10}\left(\hat{b}_{j}^{(k)}-b_{j}\right)$.

The second criterion for evaluating the item parameter estimates was based on the distance $\delta$ between each estimated curve and the corresponding true curve on the $[-3,3]$ interval divided by the length of the interval. For the $k$ th replication,

$\delta^{(k)}=\left\{\frac{1}{6} \int_{-3}^{3}\left[\hat{P}_{j k}(t)-P_{j}(t)\right]^{2} d t\right\}^{1 / 2}$,

where $P_{j}(\cdot)$ was computed using the simulation parameters and $\hat{P}_{j k}(\cdot)$ was computed with parameters estimated from the $k$ th replication. Equation 10 was evaluated using Simpson's rule and 51 grid points.

The mean $\delta^{(k)}$ across the 10 replications provided a measure of the average recovery of the item characteristic curve (ICC). It has been argued that this distance index provides the most useful measure of overall estimation accuracy because "an ICC computed from estimated item parameters could be very close to the ICC computed from actual item parameters despite large errors of estimation for $a$ and $b$ " (Hulin et al., 1982, p. 250; see also Linn, Levine, Hastings, \& Wardrop, 1981).

\section{Evaluation of Estimated Standard Errors}

To evaluate the accuracy of estimated standard errors, the true sampling variances of the parameter estimates were estimated by the observed variances

$\operatorname{Var}\left(\hat{a}_{j}\right)=\frac{1}{9} \sum_{k=1}^{10}\left(\hat{a}_{j}^{(k)}-\bar{a}_{j}\right)^{2}$

and

$\operatorname{Var}\left(\hat{b}_{j}\right)=\frac{1}{9} \sum_{k=1}^{10}\left(\hat{b}_{j}^{(k)}-\bar{b}_{j}\right)^{2}$,

where $\bar{a}_{j}$ and $\bar{b}_{j}$ are means over the 10 replications. These variances were called observed variances and their square roots were called observed standard errors (SES). The observed SEs were compared to the square roots of the average (over replications) sampling variance obtained from the Fletcher-Powell weight matrix for MMLEs. The observed SES were also compared to the square roots of the average sampling variances obtained from formulas given by Lord (1980, p. 191) for JMLEs. Standard errors obtained from Lord's formulas and from the Fletcher-Powell weight matrix were called estimated SES.

\section{Results}

\section{Item Parameter Estimates}

Mean distances between true and recovered ICCs for selected items are shown in Figure 1. The results for items 1, 3, and 4 were similar, so only the results for items 1 and 3 are presented in Figure 1 and in subsequent figures. The results for items 2,8 , and 10 are also omitted because their results were similar to other items. (The results for all the items are available from the author.)

The solid bars in Figure 1 depict results for JMLES and the remaining bars give results for MMLEs. 


\section{Figure 1}

Mean Distance Between True and Estimated Item Characteristic Curves

\section{Item 1 Item $3 \quad$ Item 5 Item 6 Item 7 Item 9}
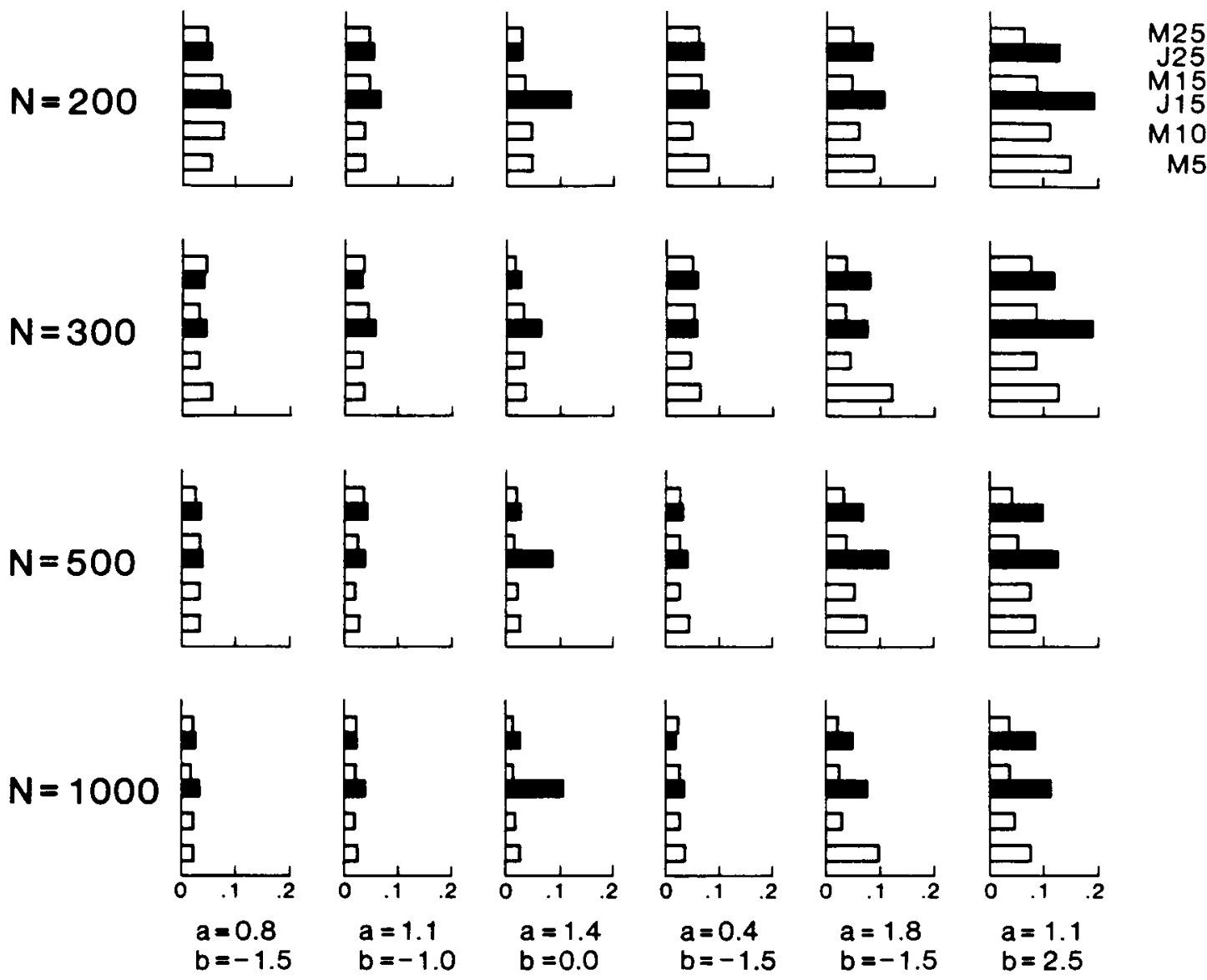

The results for MML estimation with tests of 5 items are labeled M5, results for JML estimation with tests of 15 items are labeled $\mathrm{J} 15$, and other results are labeled similarly.

The ICCs for items 1, 3, and 5 were well-recovered by MML estimation, even with the smallest sample size $(N=200)$ and shortest test $(n=5)$. For these three items the accuracy of the MMLEs was increased by increasing sample size; increasing test length had relatively modest effects.

Figure 1 shows much less satisfactory results for items 7 and 9. The ICCs for these items were not well estimated for the 5-item tests even when $N=1,000$. However, for this sample size the ICCs were well recovered by MML estimation for tests of 10 or more items. A surprising result was the accuracy of the MMLEs of the ICCs for items 7 and 9 when $n=25$ and $N=200$. In contrast, the JMLES of the ICCS for these items were generally inadequate for both 15 - and 25-item tests and all sample sizes.

The MML estimation results for item 6 are between the well-estimated items (items 1,3, and 5) and the more problematic items (items 7 and 9). The accuracy of the MMLEs of the ICC for item 6 was not greatly affected by test length, but a substantial effect for sample size is shown. Figure 1 shows that 
JMLES were reasonably satisfactory for all conditions for item 6. In fact, JML estimation for item 6 was clearly more successful than JML estimation for item 5 . This result suggests that JML estimation is relatively more successful at estimating the extreme $b$ parameter for item 6 than the extreme $a$ parameter for item 5.

The absolute values of the biases of estimates of the $a$ and $b$ parameters are shown in Figure 2, which is an adaptation of Thissen and Wainer's (1986) "XTREE glyph." Arrowheads have been placed on bars when the absolute bias was larger than the maximum value (.50) allowed in the figure. The bias of $\hat{a}$ is plotted on the left of each vertical axis, and the bias of $\hat{b}$ is plotted on the right.

Figure 2 shows that the biases of MMLEs of the $a$ and $b$ parameters for items 1,3 , and 5 are quite small. In conjunction with Figure 1, the positive results for these items show that MML estimation can be used with short unidimensional attitude scales and small to moderate samples when the magnitudes of $a$ and $b$ parameters are not too extreme.

Figure 2

Absolute Values of the Bias of Item Parameter Estimates

Item 1 Item $3 \quad$ Item 5 Item $6 \quad$ Item $7 \quad$ Item 9

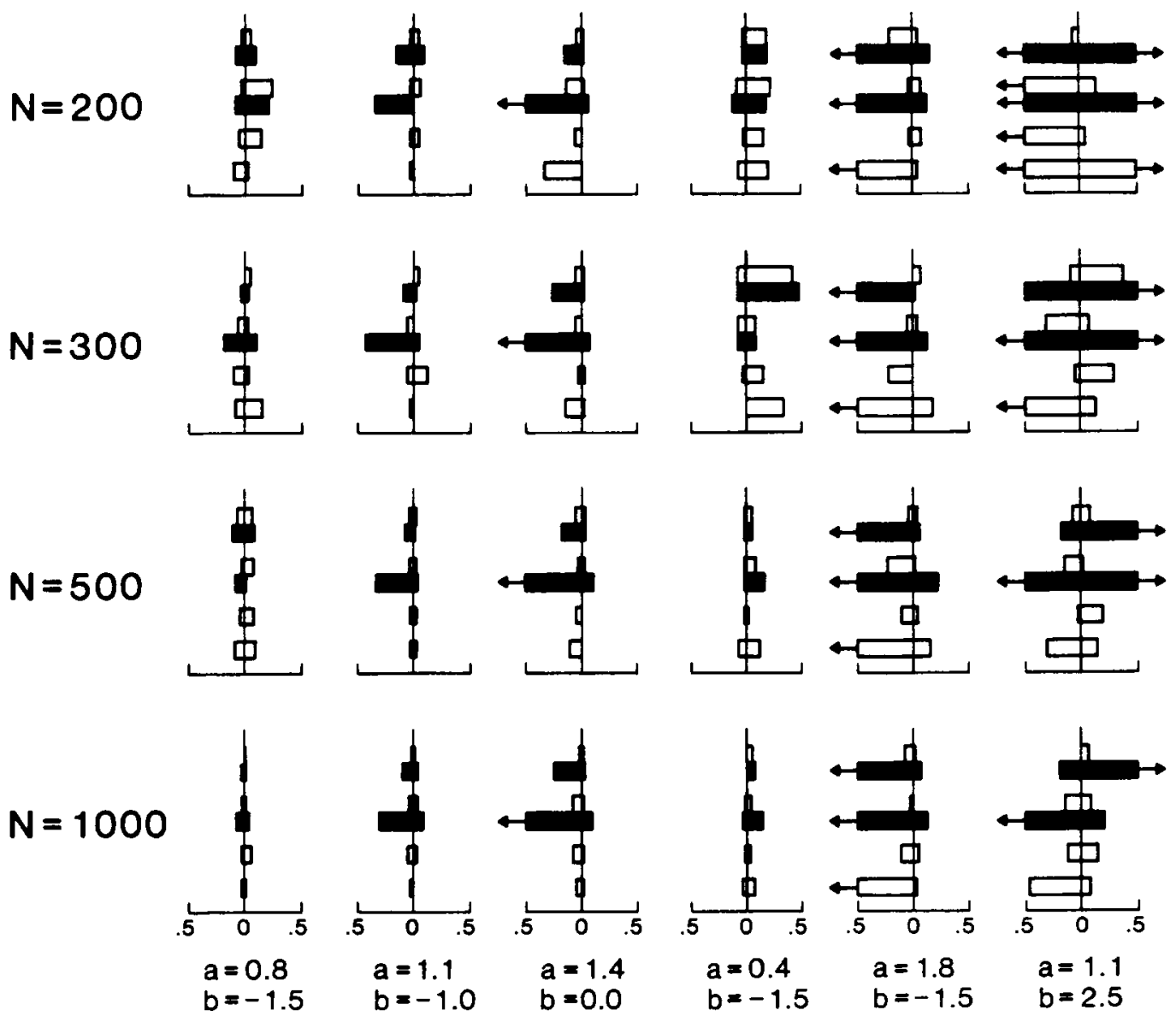


JMLEs of the $a$ parameter exhibited considerable bias for items 3 and 5 for the 15 -item test. Better results were obtained for the 25-item test, but MML estimation was clearly superior to JML estimation. Substantial amounts of bias were found for both JMLES and MMLEs of the $a$ and $b$ parameters for items 7 and 9. For MML estimation, samples of at least $N=500$ and test lengths of at least 10 items seem to be required.

Item 6 again appears to be intermediate between items 1,3 , and 5 and the more extreme items 7 and 9. Both MML and JML estimation were nearly unbiased when $N=500$ or $N=1,000$. Comparing item 5 to item 6 shows a very large (positive) bias for JMLEs of $a$ on item 5. JMLEs of $b$ were far less biased for item 6 .

\section{Estimates of Standard Errors}

Figures 3 and 4 summarize the results for observed SES of item parameter estimates, estimated SES for JMLEs obtained from Lord's (1980) formulas, and estimated SEs for the MMLEs obtained from the Fletcher-Powell weight matrix. Again, solid bars depict results for JML estimation and the remaining bars show results for MMLEs. The observed SE for each condition is plotted directly above the corresponding mean estimated SE so that poor estimates are apparent. For example, with JML estimation, $N=200$, and the 15 -item test, the mean estimated SE of the $a$ parameter for item 3 (shown in Figure 3 ) is much smaller than the observed SE.

Figures 3 and 4 show reasonably good agreement between estimated and observed SES for MMLES of both the $a$ and $b$ parameters. Sometimes both observed and estimated SEs are large (e.g., items 7 and 9), which indicates that parameter estimates were not very accurate. Sometimes both observed and estimated SEs were small, which indicates accurate estimates (when the bias is small). The important point is that no consistent tendency existed for the estimated SES for MML estimation to overestimate or underestimate the observed SES.

One qualification applies to the above statement concerning estimated SES: Estimated SES greater than 1 tended to be quite inaccurate. Evidently, the asymptotic relation that underlies estimation of SES from the Fletcher-Powell weight matrix (i.e., the relation of information to the asymptotic variance of a MLE) fails to hold when very little information is present in the data.

In contrast to the relatively accurate estimation of SES for MML estimation, the estimated SES for JML estimation are shown to be generally too small in Figures 3 and 4. Surprisingly, the observed SES were sometimes more than twice as large as the estimated SES even when the estimated SEs were as small as .05 to .10 .

\section{Estimation Accuracy Averaged Across Items}

Table 2 presents mean distances between true and estimated ICCs obtained by averaging across replications and the items presented in Figures 1 through 4. Table 2 shows that when sample size is held constant (i.e., when looking down a column of Table 2), JML estimation with 15 items is less accurate than MML estimation with 5 items. The accuracy of JML estimation with 25 items is intermediate between the accuracies of MML estimation with 5 and 10 items. Clearly, MML estimation provides accurate recovery of ICCs for much shorter tests and scales than JML estimation.

MML and JML estimation can also be compared in terms of corresponding sample sizes for tests of a given length. Table 2 shows that MML estimation with $N=200$ is more accurate than JML estimation with $N=1,000$ for the 15 -item test. For the 25-item test, JML estimation with $N=500$ is as accurate as MML estimation with $N=200$, and JML estimation with $N=1,000$ appears as accurate as MML estimation with roughly 400 examinees. 
Figure 3

Observed Standard Errors (O) and Mean Estimated Standard Errors (E) for $a$, Item 1 Item $3 \quad$ Item 5 Item 6 Item 7 Item 9
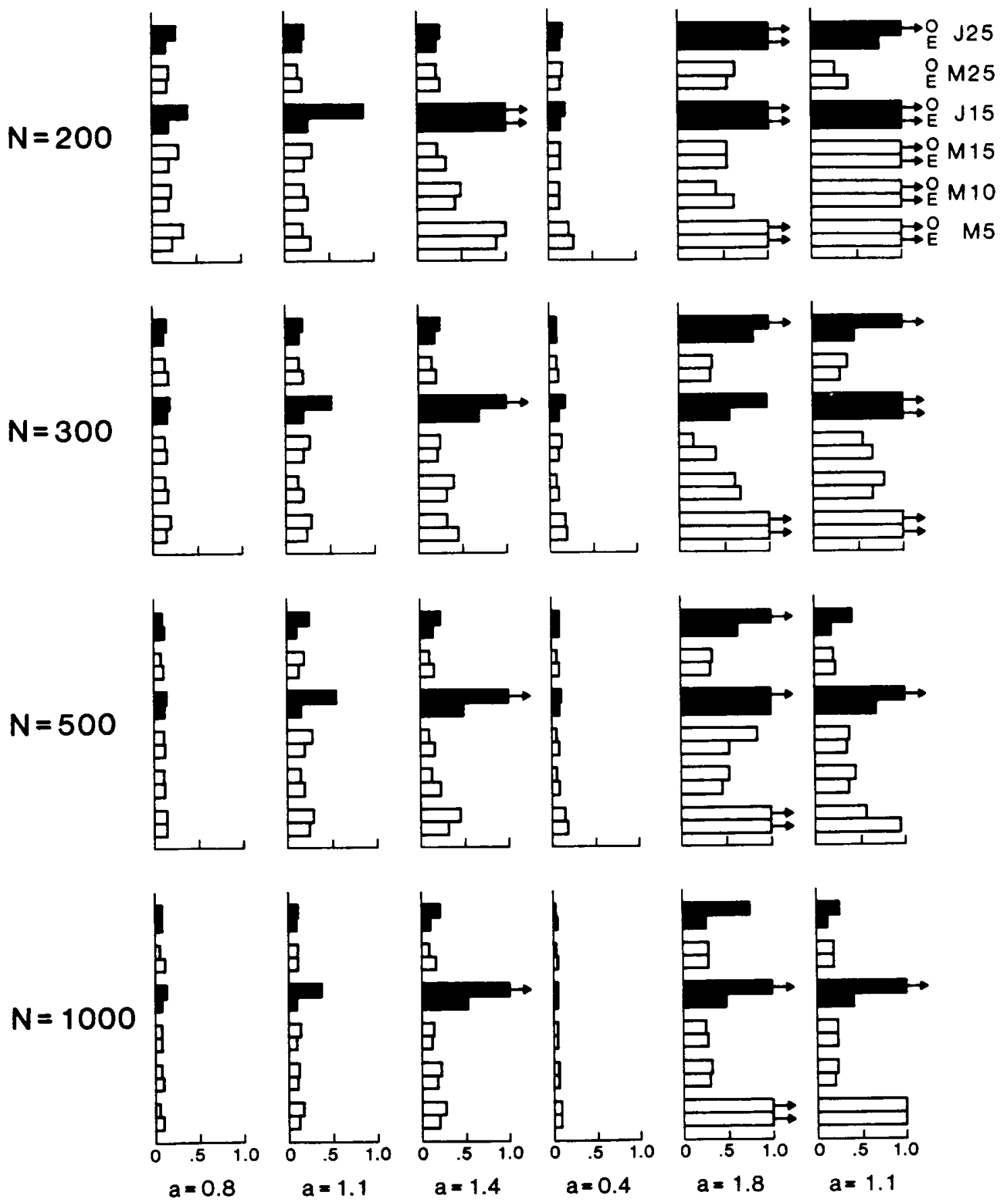
Figure 4

Observed Standard Errors (O) and Mean Estimated Standard Errors (E) for $b_{j}$ Item 1 Item $3 \quad$ Item 5 Item 6 Item 7 Item 9
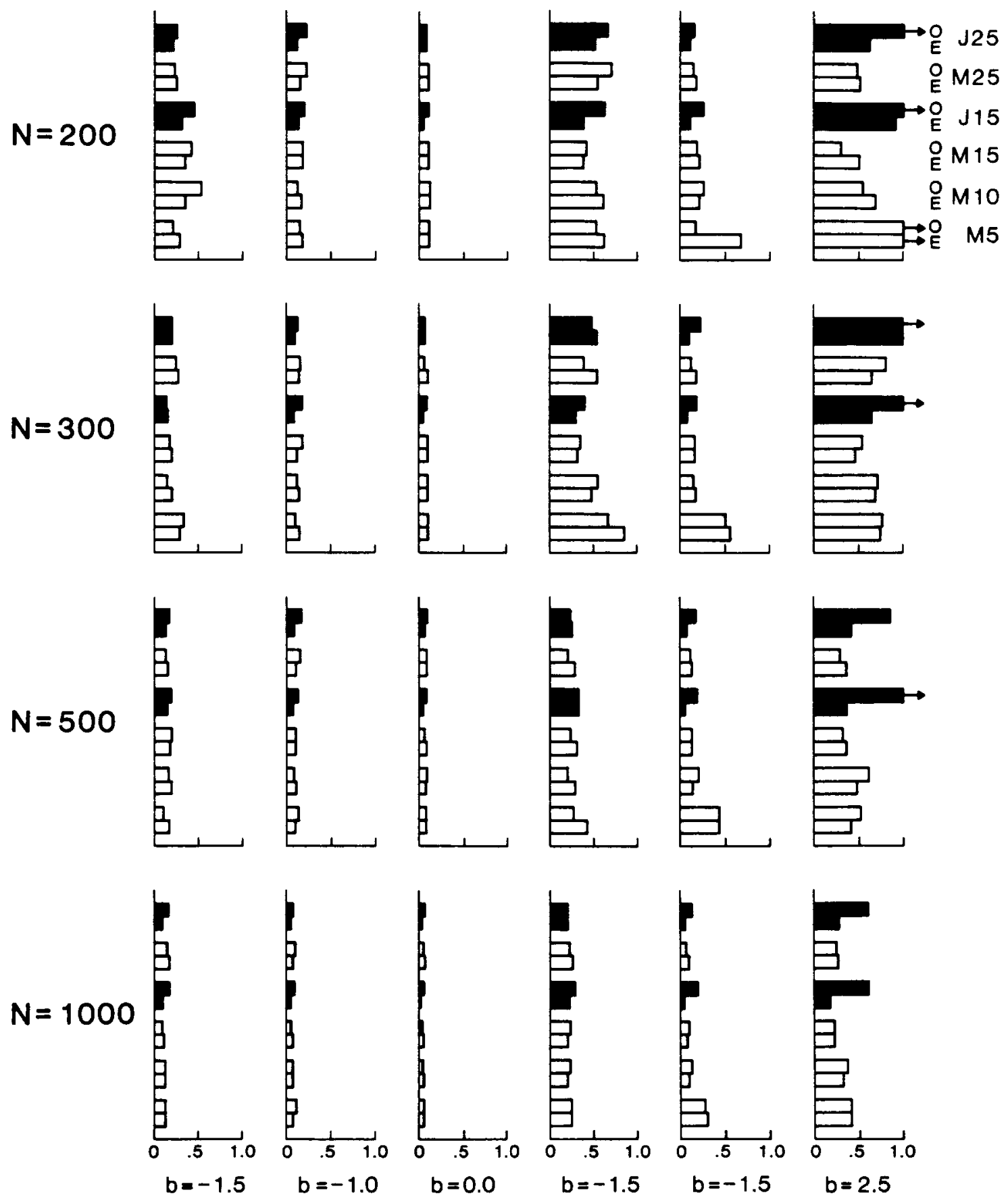
Table 2

\begin{tabular}{|c|c|c|c|c|c|}
\hline \multicolumn{6}{|c|}{$\begin{array}{l}\text { Mean Distances Between True and Estimated ICCs, } \\
\text { Averaged Over Items } 1,3,5,6,7 \text {, and } 9 \text {, } \\
\text { As a Function of Test Length and Sample Size, } \\
\text { for MML and JML Estimation }\end{array}$} \\
\hline \multirow{2}{*}{$\begin{array}{r}\text { Type of } \\
\text { Estimate }\end{array}$} & \multirow{2}{*}{$\begin{array}{c}\text { Test } \\
\text { Length }\end{array}$} & \multicolumn{4}{|c|}{ Sample Size } \\
\hline & & 200 & 300 & 500 & 1,000 \\
\hline MML & 5 & .074 & .074 & .048 & .049 \\
\hline MML & 10 & .062 & .046 & .037 & .028 \\
\hline MML & 15 & .057 & .048 & .031 & .024 \\
\hline JML & 15 & .104 & .082 & .073 & .066 \\
\hline MML & 25 & .046 & .043 & .030 & .023 \\
\hline JML & 25 & .066 & .058 & .049 & .038 \\
\hline
\end{tabular}

\section{Discussion}

These results illustrate the range of conditions that allow accurate calibration of two-parameter logistic items by MML. For items with parameters typical of some attitude scales (namely, items 1 through 5 in this simulation), as few as 200 persons and 5 items are required for essentially unbiased parameter estimates with reasonably small SES. Furthermore, the algorithm used here to maximize the likelihood function provided estimated SEs that were in close agreement with observed SEs.

The parameters of items 6 through 10 were selected to correspond to extreme items. In fact, the 5item scale formed by these items represents a worst-case scenario; most items from one important scale (the JDI) have easily estimated parameters (i.e., items 1 through 5), and only a few items have extreme parameters. Thus, the test composed of items 6 through 10 corresponded to a situation where a researcher has selected only extreme items instead of a more reasonable mix.

The analyses of items 6 though 10 show the types of difficulties that can be encountered with MML estimation: Estimates of large $a$ parameters have positive biases and large SES, and estimates of $b$ parameters large in magnitude are biased and have large SEs. Figures 3 and 4 show that the Fletcher-Powell SES will ordinarily inform researchers that extreme parameter estimates are inaccurate. For example, the appropriate interpretation of an $\hat{a}=5$ with a Fletcher-Powell SE of 8 is that the item is discriminating, but its parameters could not be estimated from the dataset by the method of MML.

JMLES were much less accurate than MMLEs, and the estimated SES of JMLEs were far less accurate than the Fletcher-Powell SEs of MMLEs. In fact, the estimated SEs from JML estimation could seriously mislead researchers in some situations.

The EM algorithm provides an alternative approach to obtaining MMLEs. It maximizes Equation 4 much more quickly than the Fletcher-Powell algorithm because it does not use first derivatives computed from the responses of individual examinees. Fletcher-Powell iterations require such first derivatives (and they involve extensive computations). Unfortunately, the EM algorithm does not produce SEs for parameter estimates. Of course, all the elements in the $2 n \times 2 n$ matrix of observed second derivatives could be evaluated after maximizing the likelihood function with the EM algorithm. However, computing all of the second derivatives once for a 15-item test would involve more computations than the entire function maximization by the Fletcher-Powell algorithm (assuming 25 iterations). As an alternative to evaluating the entire $2 n \times 2 n$ matrix of second derivatives, the $2 \times 2$ submatrices of second derivatives for each item might be used to approximate SEs. The accuracy of this approach apparently has not yet been studied carefully. Given the problems with estimated SEs for JMLES, it seems clear that any method selected for estimating SES should be carefully evaluated. 
The accuracy of estimation varied greatly across the items used in this simulation, suggesting that estimation accuracy in studies that average across items (e.g., Hulin et al., 1982) depends greatly on the values of the particular item parameters selected. In this study, estimation accuracy was evaluated for individual items through the use of replications. The limitations on generalizability may be less severe with the approach used here, although accuracy for a given item certainly depends on the other $n-1$ items in the test. Thus, the generality of these results is limited by the specific item parameters used in the simulation and, in particular, the asymmetry of the distribution of item difficulties. Nonetheless, comparisons across studies by different researchers using different estimation methods would be facilitated if results were reported for individual items.

Finally, the accuracy of estimates of the parameters of items 6 through 10 of this study could be improved by using appropriate Bayesian prior distributions. The Bayesian methods developed by Swaminathan and Gifford (1985) and Tsutakawa and Lin (1986) for the two-parameter logistic model should be very useful. One issue that should be investigated, however, involves the effects of the statistical bias of Bayes estimates. For example, what will be the effects of this bias in the context of an item bias study? Although Bayesian methods should reduce SES of parameter estimates, their effects on the ability to detect item bias and avoid Type I errors are less clear.

\section{References}

Bock, R. D., \& Aitkin, M. (1981). Marginal maximum likelihood estimation of item parameters: Application of an EM algorithm. Psychometrika, 46, 443-459.

Bock, R. D., \& Lieberman, M. (1970). Fitting a response model for $n$ dichotomously scored items. Psychometrika, 35, 179-197.

Dempster, A. P., Laird, N., \& Rubin, D. B. (1977). Maximum likelihood from incomplete data via the EM algorithm (with discussion). Journal of the Royal Statistical Society, Series B, 39, 1-38.

Drasgow, F., \& Hulin, C. L. (in press). Cross-cultural measurement. Interamerican Journal of Psychology.

Fletcher, R., \& Powell, M. J. D. (1963). A rapidly convergent descent method for minimization. Computer Journal, 2, 163-168.

Gruvaeus, G. T., \& Jöreskog, K. G. (1970). A computer program for minimizing a function of several variables (Research Bulletin 70-14). Princeton NJ: Educational Testing Service.

Hulin, C. L. (1987). A psychometric theory of evaluations of item and scale translation: Fidelity across languages. Journal of Cross-Cultural Psychology, 18, $115-142$.

Hulin, C. L., Drasgow, F., \& Parsons, C. K. (1983). Item response theory: Application to psychological measurement. Homewood IL: Dow Jones-Irwin.

Hulin, C. L., Lissak, R. I., \& Drasgow, F. (1982). Recovery of two- and three-parameter logistic item characteristic curves: A monte carlo study. Applied Psychological Measurement, 6, 249-260.

Kiefer, J., \& Wolfowitz, J. (1956). Consistency of the maximum likelihood estimator in the presence of infinitely many incidental parameters. Annals of Mathematical Statistics, 27, 887-906.

Linn, R. L., Levine, M. V., Hastings, C. N., \& Wardrop, J. L. (1981). Item bias in a test of reading comprehension. Applied Psychological Measurement, $5,159-173$.

Lord, F. M. (1968). An analysis of the Verbal Scholastic Aptitude Test using Birnbaum's three-parameter logistic model. Educational and Psychological Measurement, 28, 989-1020.

Lord, F. M. (1980). Applications of item response theory to practical testing problems. Hillsdale NJ: Erlbaum.

Lord, F. M., \& Novick, M. R. (1968). Statistical theories of mental test scores. Reading MA: AddisonWesley.

McLaughlin, M. E., \& Drasgow, F. (1987). Lord's chisquare test of item bias with estimated and with known person parameters. Applied Psychological Measurement, 11, 161-173.

Mislevy, R. J. (1986). Bayes modal estimation in item response models. Psychometrika, 51, 177-195.

Mislevy, R. J., \& Bock, R. D. (1984). Item operating characteristics of the Armed Services Vocational Aptitude Battery (ASVAB) Form 8A. Chicago: National Opinion Research Center.

Mislevy, R. J., \& Bock, R. D. (1985). Implementation of the EM algorithm in the estimation of item parameters: The BILOG computer program. In D. J. Weiss (Ed.), Proceedings of the 1982 Item Response Theory and Computerized Adaptive Testing Conference. Min- 
neapolis: University of Minnesota, Department of Psychology, Computerized Adaptive Testing Laboratory.

Neyman, J., \& Scott, E. L. (1948). Consistent estimates based on partially consistent observations. Econometrika, 16, 1-32.

Smith, P. C., Kendall, L. M., \& Hulin, C. L. (1969). The measurement of satisfaction in work and retirement. Skokie IL: Rand McNally.

Swaminathan, H., \& Gifford, J. A. (1985). Bayesian estimation in the two-parameter logistic model. Psychometrika, 50, 349-364.

Thissen, D. (1982). Marginal maximum likelihood estimation for the one-parameter logistic model. Psychometrika, 47, 175-186.

Thissen, D. (1986). MULTLOG user's manual. Mooresville IN: Scientific Software.

Thissen, D., \& Steinberg, L. (1984). A response model for multiple choice items. Psychometrika, 49, 501519.
Thissen, D., \& Wainer, H. (1986). XTREE: A multivariate graphical icon applicable in the evaluation of statistical estimators. American Statistician, 40, 149153.

Tsutakawa, R. K., \& Lin, H. Y. (1986). Bayesian estimation of item response curves. Psychometrika, 51 , 251-267.

Wingersky, M. S., Barton, M. A., \& Lord, F. M. (1982). LOGIST user's guide. Princeton NJ: Educational Testing Service.

\section{Author's Address}

Send requests for reprints or further information to Fritz Drasgow, Department of Psychology, University of Illinois, 603 E. Daniel Street, Champaign IL 61820, U.S.A. 